\title{
Forecasting telecommunications data with linear models
}

\author{
Gary Madden ${ }^{\mathrm{a}, *}$, Joachim $\operatorname{Tan}^{\mathrm{b}}$ \\ ${ }^{a}$ School of Economics and Finance, Curtin University of Technology, GPO Box U1987, Perth, WA 6845, Australia \\ ${ }^{\mathrm{b}}$ School of Economics and Finance, Curtin University of Technology, Perth, WA 6845, Australia
}

\begin{abstract}
For telecommunication companies to successfully manage their business, companies rely on mapping future trends and usage patterns. However, the evolution of telecommunications technology and systems in the provision of services renders imperfections in telecommunications data and impinges on a company's' ability to properly evaluate and plan their business. International Telecommunication Union (ITU) Recommendation E.507 provides a selection of econometric models for forecasting these trends. However, no specific guidance is given. This paper evaluates whether simple extrapolation techniques in Recommendation E.507 can generate accurate forecasts. Standard forecast error statistics - mean absolute percentage error (MAPE), median absolute percentage error and percentage better-show the ARIMA, Holt and Holt-D models provide better forecasts than a random walk and other linear extrapolation methods.
\end{abstract}

(C) 2006 Elsevier Ltd. All rights reserved.

Keywords: Linear models; ITU Recommendations; Telecommunications forecasting

\section{Introduction}

In the current environment telecommunications industry boundaries are rapidly changing, markets are increasingly competitive, and company-based data are more fragmented and proprietary. Within this context, telecommunications companies must develop strategy to deal with a rapidly changing and expanding mix of telecommunications services, especially the emergence of substitutes and complements. That is, to successfully manage their business carriers must rely on data to monitor, analyze and optimize their systems to map future trends and use patterns. Forecasting is an integral input into such network traffic management, infrastructure optimization and planning, and the scenario planning process. Data-based forecasts, excluding the analysis, are constructed from data that is processed via statistical models from which inferences are drawn. The analysis of Grubesic and Murray (2005) addresses concerns related to the quality of available (spatial) data. In particular, Grubesic and Murray argue that, because of the complexity of telecommunications systems, analysts need to concern themselves with the impact of using imperfect spatial information. Finally, Grubesic

\footnotetext{
*Corresponding author. Tel.: + 61892664258 ; fax: + 61892662391.

E-mail address: gary.madden@cbs.curtin.edu.au (G. Madden).
} 
and Murray develop a framework to address the sensitivity of spatial analyzes to imperfect spatial data. By contrast, this study focuses on identifying appropriate statistical method given no information about the available data. This approach is in the spirit of the International Telecommunication Union (ITU) Recommendation E.507. The Recommendation provides an overview of existing mathematical techniques for forecasting that includes curve-fitting models, autoregressive models, autoregressive integrated moving average (ARIMA) models, state space models with Kalman filtering and regression models. ${ }^{1}$ However, Recommendation E.507 provides no guidance as to which models are the most appropriate for forecasting telecommunications series. This study contributes by examining the forecast performance of these extrapolation models by comparing forecasts against those from a random walk model. ${ }^{2}$ In particular, the paper follows the format of a competition between simple models that do not require detailed domain knowledge. Namely, the comparison of forecast accuracy is based on telecommunications data via series pattern recognition. The paper is structured as follows. Section 2 describes the M3-competition telecommunications data. Section 3 reviews linear univariate forecast methods used in this study, and discusses error statistics used to evaluate forecast performance. Section 4 presents the forecast results and Section 5 concludes.

\section{Data}

Data used are acquired from the Institute of Forecasters web site located at http://www.forecasters.org. M3-competition data are comprised of 3003 series. One hundred and forty nine series are telecommunications industry data, of which 29 are monthly series and 120 series are of unknown periodicity (and labelled 'other'). Monthly and 'other' series consist of 53 and 63 observations, respectively. All observations have strictly positive values. No additional information is provided by the Institute. A representative specimen of the monthly and 'other' data are shown in Figs. 1 and 2.

Grambsch and Stahel (1990) and Fildes (1992) find telecommunications data exhibit both non stationary and strong negative trends. ${ }^{3}$ Similarly, most of the monthly and 'other' series in this study exhibit negative trends. For the monthly series, only one of 29 series shows a positive trend. For the 'other' series, 115 series exhibits a negative trend, while only five series exhibit a positive trend.

Average summary statistics for the monthly and 'other' series are presented in Tables 1 and 2, respectively. The tables indicate that the mean, standard deviation, degree of skewness and number of outliers for the 'other' series are higher than those of the monthly series. The sample average of standard deviation for the monthly and 'other' series are 386.8 and 921.5, respectively. This indicates that the spread of the 'other' series is about 2.4 times larger than that of the monthly series. The corresponding coefficients of variation $(0.08$ and 0.13 ) for both series also indicate the differences in the spread of the series.

Following Fildes (1992), frequency of outliers, strength of trend and degree of randomness for the data are analyzed. Results are shown in Figs. 5 and $6 .^{4}$

Fig. 3 reveals 26 of the 29 monthly series contain only a single outlier, with three series containing more than one outlier. The 'other' series exhibit some similar properties. That is Fig. 4 shows that 116 of the 120 'other'

\footnotetext{
${ }^{1}$ Recommendation E.507 also describes methods to evaluate and select an appropriate technique, depending on available data and forecast period.

${ }^{2}$ Standard univariate linear models are useful for this application as they are simple to implement and easily understood, and therefore used for commercial applications.

${ }^{3}$ These observations lead Grambsch and Stahel (1990) and Fildes (1992) to argue the simple exponential smoothing model is inappropriate for forecasting these data.

${ }^{4}$ An observation is defined as being an outlier when either $X_{t}<L_{x}-1.5\left(U_{x}-L\right)$ or $X_{t}>L_{x}+1.5\left(U_{x}-L\right)$, where $L_{x}$ denotes the lower quartile and $U_{x}$ is the upper quartile. The strength of trend is measured by the correlation between series (with outliers removed) and a time trend, with the absolute value of the trend indicating its strength. Randomness is measured by estimating the regression:

$$
X_{t}^{\prime}=\alpha+\beta t+\delta_{1} X_{t-1}^{\prime}+\delta_{2} X_{t-2}^{\prime}+\delta_{3} X_{t-3}^{\prime},
$$

where $X_{t}^{\prime}$ denotes the series Xt with outliers removed. $\bar{R}^{2}$ measures the variation explained by the model. A higher $\bar{R}^{2}$ indicates little randomness, while a relatively low $\bar{R}^{2}$ suggests a higher degree of randomness.
} 


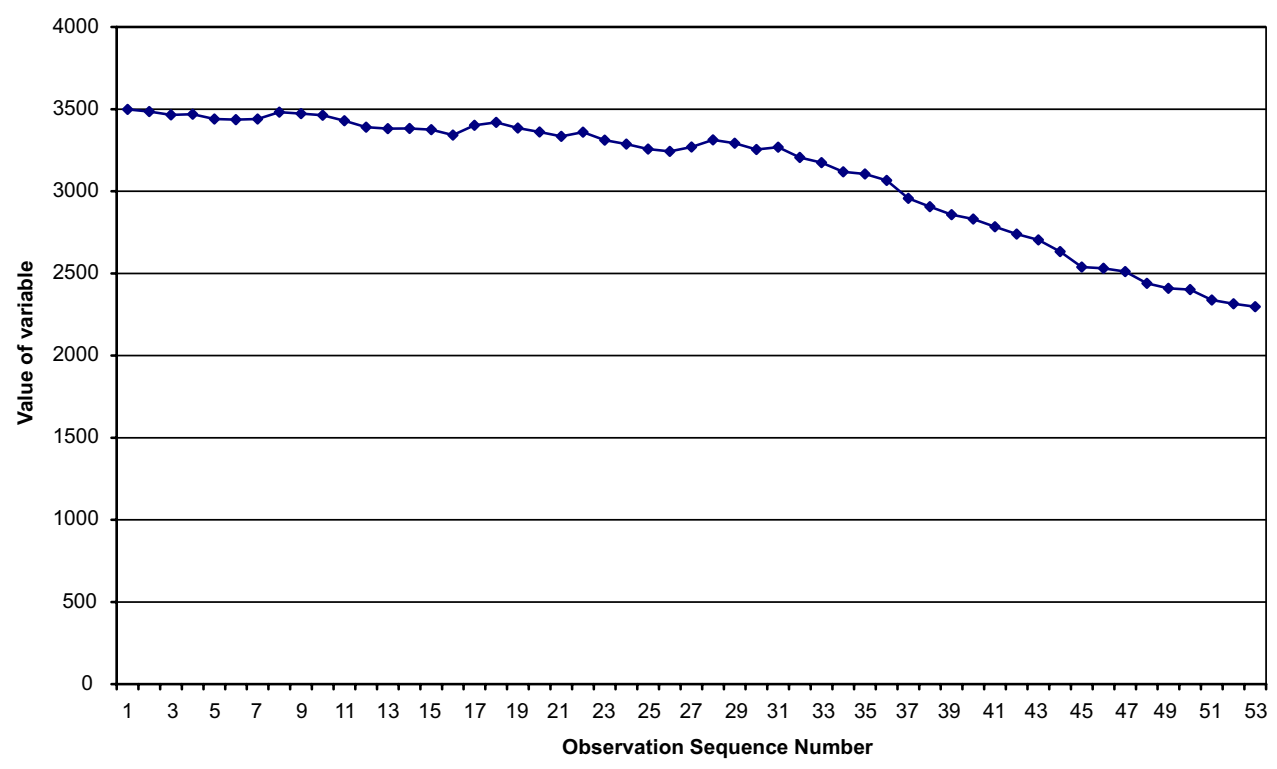

Fig. 1. Specimen of a M3 monthly series; Source. IIF(2005).

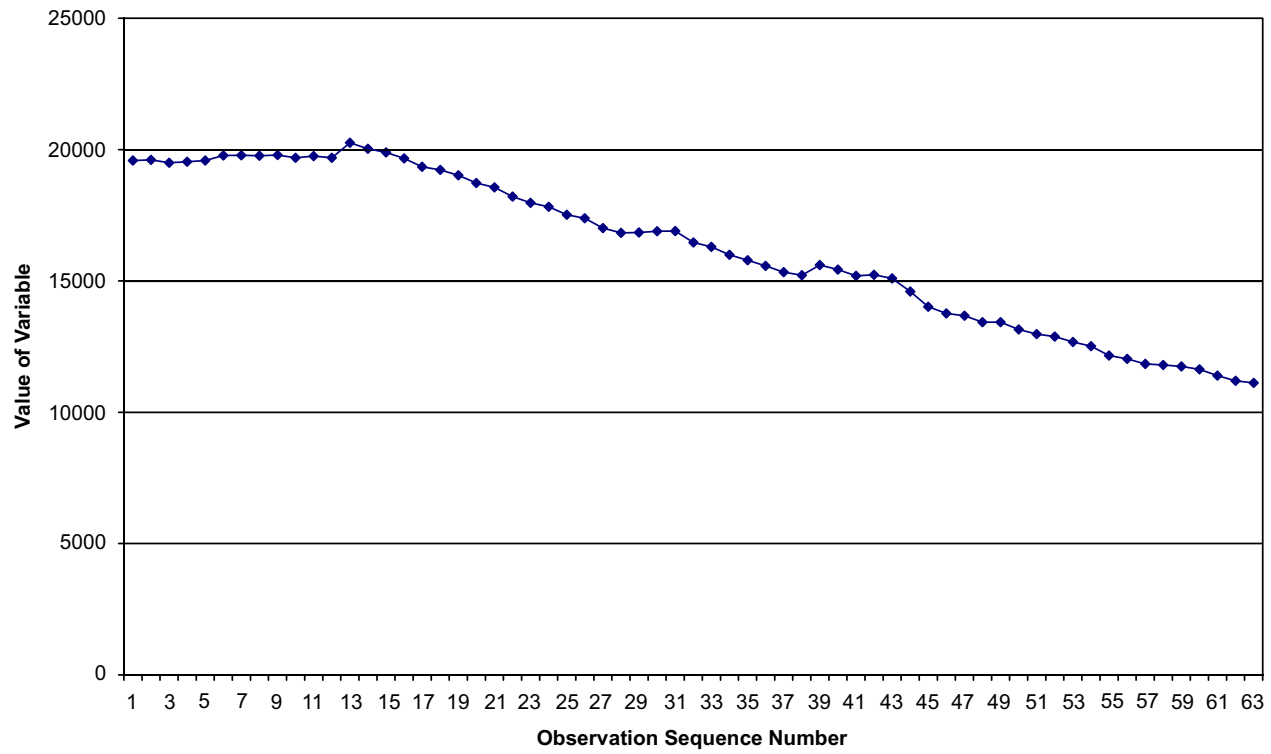

Fig. 2. Specimen of a M3 'Other' series; Source. IIF(2005).

series contain only one outlier, two series contain 11 outliers and two series contains 14 outliers. The characteristics of the M3 telecommunications data appear to be homogenous and have similar properties to the telecommunications data analyzed by Fildes (1992).

Figs. 5 and 6 show the strength of the trend for the M3 data. The monthly series have the stronger negative correlation with time. The histograms contained in Figs. 5 and 6 also reveal a higher correlation for the monthly series. 
Table 1

Summary statistics for M3 monthly series

\begin{tabular}{|c|c|c|c|c|c|c|}
\hline & Mean & Variance & Skewness & Kurtosis & Minimum & Maximum \\
\hline Mean & $4,953.84$ & $2,337.83$ & 0.88 & 2.61 & $2,183.06$ & $10,740.60$ \\
\hline Variance & 386.80 & 362.29 & 2.55 & 10.37 & 57.75 & $1,867.98$ \\
\hline Skewness & 73.91 & 36.66 & 0.07 & 1.74 & 11.04 & 134.92 \\
\hline Kurtosis & -0.82 & 0.85 & 1.84 & 6.35 & -1.90 & 2.13 \\
\hline Turning Points & 10.00 & 3.09 & -0.54 & 2.11 & 4.00 & 15.00 \\
\hline Step Changes & 0.48 & 0.87 & 1.51 & 3.89 & 0.00 & 3.00 \\
\hline Outliers & 27.97 & 6.63 & -3.14 & 11.42 & 2.00 & 30.00 \\
\hline $\mathrm{CV}$ & 0.08 & 0.08 & 3.29 & 14.81 & 0.02 & 0.46 \\
\hline Maximum & $5,562.57$ & $2,489.95$ & 0.74 & 2.58 & $2,384.60$ & $11,855.20$ \\
\hline Minimum & $4,331.32$ & $2,159.54$ & 0.78 & 2.26 & $1,879.20$ & $8,971.50$ \\
\hline Runs & 16.83 & 4.98 & -0.36 & 2.21 & 6.00 & 24.00 \\
\hline Autocorrelation & 0.95 & 0.07 & -3.11 & 12.36 & 0.65 & 0.99 \\
\hline
\end{tabular}

Note: The number of series tested is 29 . The summary statistics report represents the summary statistics of each series. Std Dev is the standard deviation; Turing Points and Step Changes are the number of turning points and step changes, respectively, defined in Shah (1997); Outliers is the number of outliers greater than three standard deviations; CV is the coefficient of variation; Runs is the number of runs; autocorrelation is the estimate of autocorrelation of lag 1.

Table 2

Summary statistics of M3 'Other' series

\begin{tabular}{|c|c|c|c|c|c|c|}
\hline & Mean & Variance & Skewness & Kurtosis & Minimum & Maximum \\
\hline Mean & $6,748.04$ & $5,988.95$ & 2.75 & 13.31 & $1,749.82$ & $42,191.30$ \\
\hline Std Dev & 921.50 & $1,315.54$ & 4.37 & 23.94 & 109.88 & $8,988.27$ \\
\hline Skewness & 102.49 & 46.78 & 0.93 & 5.92 & 3.70 & 319.65 \\
\hline Kurtosis & -0.94 & 0.82 & 4.84 & 34.51 & -1.90 & 5.49 \\
\hline Turning Points & 12.88 & 4.62 & 0.02 & 2.60 & 2.00 & 23.00 \\
\hline Step Changes & 0.57 & 1.00 & 1.70 & 4.86 & 0.00 & 4.00 \\
\hline Outliers & 48.10 & 6.68 & -3.88 & 17.25 & 15.00 & 50.00 \\
\hline $\mathrm{CV}$ & 0.13 & 0.06 & 1.03 & 4.44 & 0.03 & 0.32 \\
\hline Maximum & $8,146.49$ & $7,821.10$ & 3.14 & 15.79 & $2,127.00$ & $55,794.00$ \\
\hline Minimum & $5,221.50$ & $4,523.13$ & 2.65 & 12.67 & $1,276.00$ & $30,908.00$ \\
\hline Runs & 17.58 & 5.46 & 0.00 & 2.82 & 4.00 & 30.00 \\
\hline Autocorrelation & 0.98 & 0.03 & -4.71 & 27.37 & 0.81 & 1.00 \\
\hline
\end{tabular}

Note: The number of series tested is 120 . The summary statistics report represents the summary statistics of each series. Std Dev is the standard deviation; Turing Points and Step Changes are the number of turning points and step changes, respectively, defined in Shah (1997); Outliers is the number of outliers greater than 3 standard deviations; CV is the coefficient of variation; Runs is the number of runs; autocorrelation is the estimate of autocorrelation of lag 1.

Figs. 7 and 8 show both the monthly and 'other' series exhibit a low degree of randomness and have a strong positive correlation with a linear trend. The results suggests that it is appropriate to use linear models to forecast the M3 telecommunications data.

\section{Forecast models applied}

The univariate linear extrapolative techniques applied for forecasting are ARARMA (Parzen 1982), ARIMA, Holt, Holt-D, Holt-Winters, simple exponential smoothing (SES) and the robust trend (RT; Grambsch \& Stahel 1990) models. ${ }^{5}$ These forecast methods are used as the models are proposed in

\footnotetext{
${ }^{5}$ Only the no trend, no seasonal version of the SES model is included in the analysis, i.e., the SES model used is $y_{t}=y_{t-1}+\alpha e_{t}$, with $\alpha=0.3$.
} 


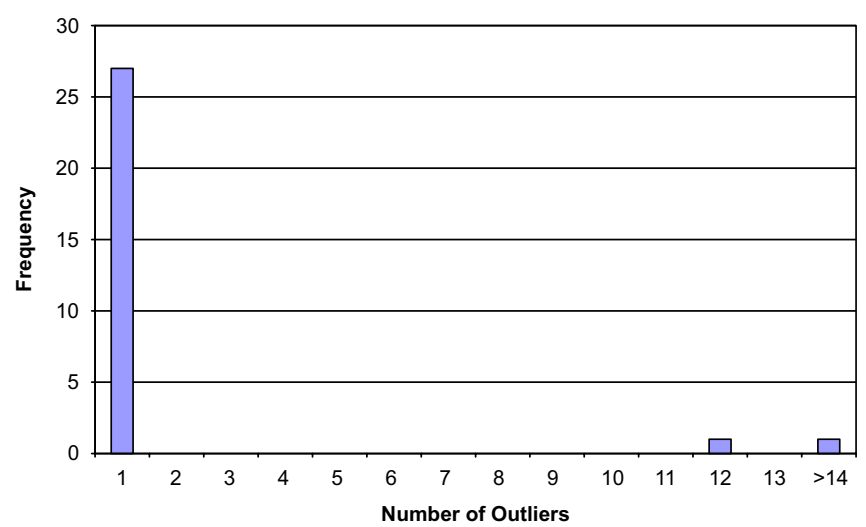

Fig. 3. Outlier frequency of M3 monthly series.

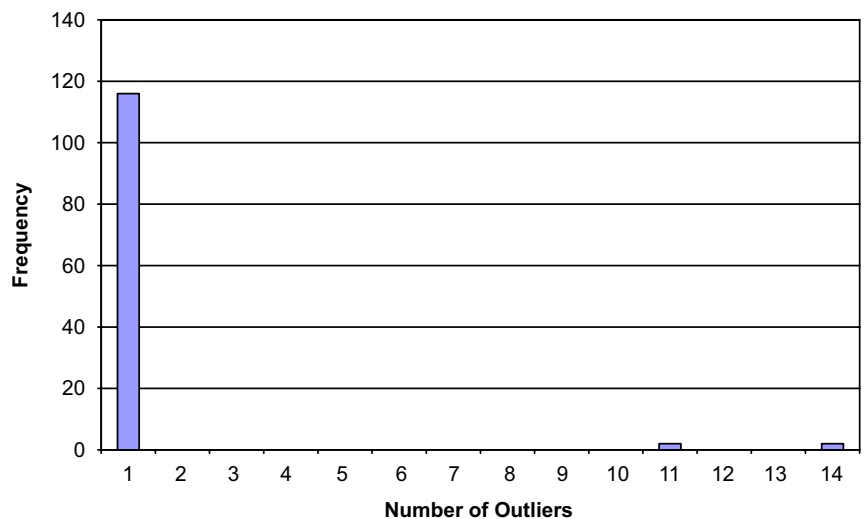

Fig. 4. Outlier frequency of M3 'Other' series.

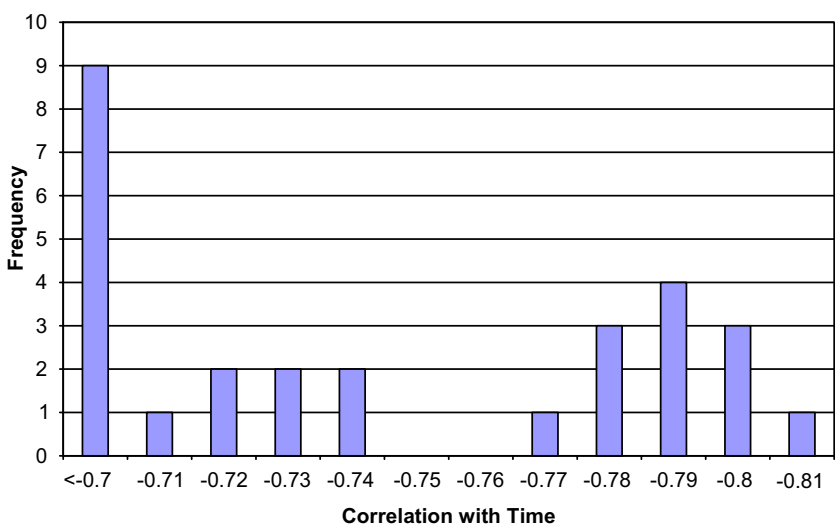

Fig. 5. Correlation with time for M3 monthly series.

Recommendation E.507 and are shown to be reliable by Makridakis et al. (1993), Fildes, Hibon, Makridakis, and Meade (1998), and Makridakis and Hibon (2000) by performing consistently in the M-competition studies. All models are estimated beginning at observation 6 . This means parameter estimates for all models are estimated from observation 6 to observation 53, and observation 6 to observation 63 for the monthly and the 'other' series, respectively. To estimate the parameters of the ARARMA and ARIMA models, an 


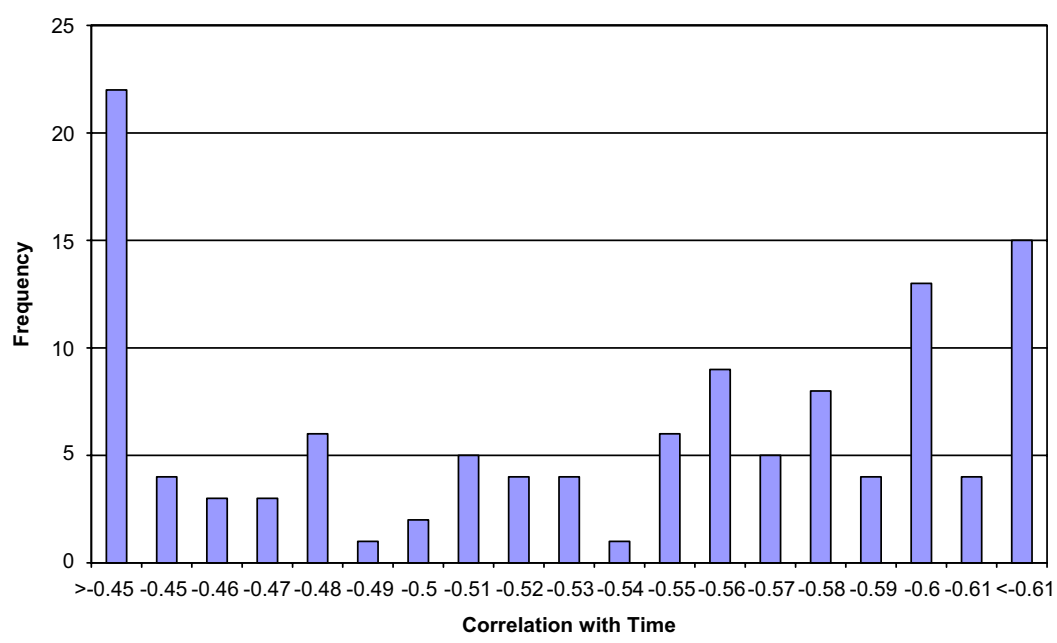

Fig. 6. Correlation with time for M3 'Other' series.

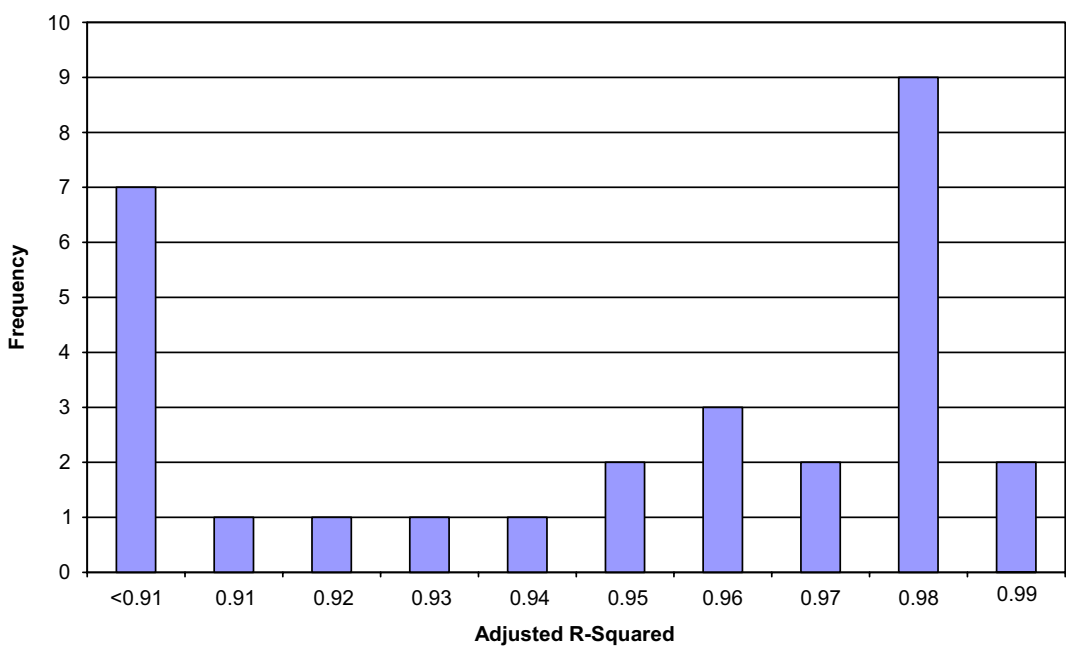

Fig. 7. Variation explained by the Linear/AR for M3 monthly series.

automatic procedure with a maximum 5-period lag is used. The automatic procedure estimates the parameters of all possible combinations of ARARMA and ARIMA models within the imposed lag limit generating 60 possible models for each ARARMA and ARIMA model, respectively. ${ }^{6}$ A grid search is then carried out on the generated ARARMA and ARIMA models to determine the optimal lag length. This is done by comparing the Akaike information criterion (AIC) statistic values of the generated models. ${ }^{7}$ The 'best' ARARMA and ARIMA models are those that generate the lowest AIC. Holt, Holt-D, Holt-Winters and RT models have

\footnotetext{
${ }^{6}$ The maximum lags for the ARARMA model is 5 periods. The ARARMA is estimated by applying an AR model 1- and 2-period lags. Residuals are estimated with another ARMA model with lags of 1- to 3-periods to generate the ARARMA model.

$$
A I C=\log \left(\hat{\sigma}_{(p, q)}^{2}\right)+\frac{2 m}{T},
$$

where $\hat{\sigma}_{(p, q)}^{2}$ is the variance of the residuals of the estimated model. T is the number of observations and $\mathrm{m}$ is the number of parameters of the univariate model tested.
} 


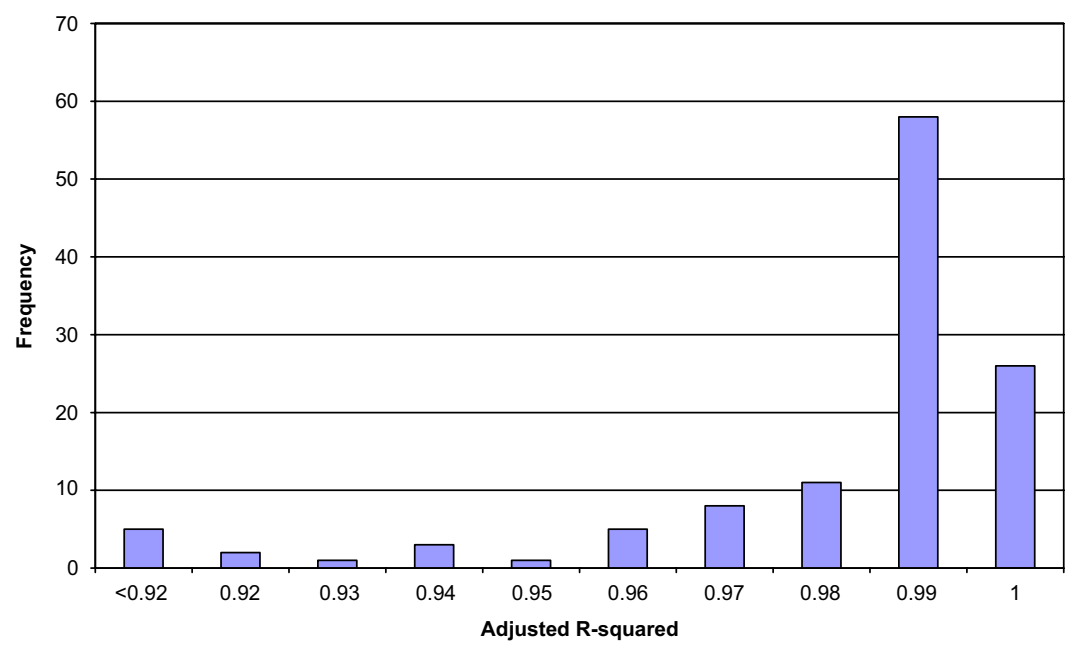

Fig. 8. Variation explained by the Linear/AR for M3 'Other' series.

their lag lengths fixed and so do not require a grid search to select best model. ${ }^{8}$ Following Makridakis and Hibon (2000), a best model - for both series and method - is used to forecast a maximum 18 observations and eight observations ahead for the first forecast in the sequence, respectively, for the monthly and 'other' series. For the second forecast in the sequence, the data series expands by one period (one-step ahead) and forecasts are made for 17 observations and seven observations ahead, respectively. Forecast accuracy measures used are guided by Armstrong and Collopy (1992), viz., mean absolute percentage error (MAPE), median absolute percentage error (MdAPE) and percent better (PB). The error statistics are defined in the Appendix. ${ }^{9}$

\section{Forecasts}

To identify the most accurate forecast model, aggregate results by method are compared using an out of sample forecast horizon for the monthly and 'other' series data. Following Makridakis and Hibon (2000), a maximum of 18 and eight steps ahead are generated for monthly and 'other' data, respectively. Forecast accuracy is compared using MAPE, MdAPE and PB error statistics. Tables 3 and 4 present the MAPE and MdAPE results for the out-of-sample monthly forecasts. MAPE statistic show the Holt model as best forecast method for medium-and long-horizons (short-, intermediate- and long-horizons are considered), as it consistently the yields lowest percentage error when compared to sample data. The MdAPE statistic indicates the Holt-D model is the best method for medium- and long-horizons. For short-horizon forecasting (1-period ahead), the MAPE and MdAPE statistics suggest the ARIMA model is best model.

The results for the PB statistic are reported for monthly series in Table 5. Similar to the MdAPE results of Table 4 and 5 shows that the ARIMA and the Holt-D model are the best. Table 5 shows that the ARIMA model is best at forecasting short- and long-horizons for 1-period, 12-periods and 18-periods ahead, while the Holt-D model is best at forecasting intermediate- to long-horizons of 6-periods and 12-periods ahead.

Figs. 9-11 illustrate forecasts for 1-period to 18-periods ahead for the best forecast methods using monthly data. The MAPE statistic in Fig. 9 shows the Holt and RW models is best for most forecast horizons. At shorter horizons of 8-periods ahead or less, the MAPE statistic shows the ARIMA and Holt models generate the best forecasts.

\footnotetext{
${ }^{8}$ Only the linear, no trend and non-seasonal versions of the Holt and Holt-W methods are considered. The Holt-D model is the exponentially smoothed version of the Holt model. Parameters for these models are estimated from data rather than being fixed arbitrarily.

${ }^{9}$ Mean square error measures are not used as they are scale dependent and sensitive to outliers.
} 
Table 3

Mean absolute percentage error for M3 monthly series

\begin{tabular}{|c|c|c|c|c|}
\hline \multirow[t]{2}{*}{ Forecast method } & \multicolumn{4}{|c|}{ Forecast horizon } \\
\hline & 1 & 6 & 12 & 18 \\
\hline RT & 0.136 & 0.182 & 0.211 & 0.235 \\
\hline ARIMA & 0.004 & 0.077 & 0.183 & 0.322 \\
\hline HOLT & 0.012 & 0.066 & 0.124 & 0.185 \\
\hline HOLT-D & 0.008 & 0.085 & 0.397 & 4.119 \\
\hline HOLT-W & 0.016 & 0.094 & 0.184 & 0.292 \\
\hline ARARMA & 0.860 & 0.949 & 1.104 & 1.815 \\
\hline SES & 0.042 & 0.119 & 0.190 & 0.267 \\
\hline RW & 0.013 & 0.087 & 0.161 & 0.236 \\
\hline
\end{tabular}

Note: RT is the robust trend model; ARIMA is the autoregressive integrated moving average model; HOLT is Holt's linear no trend model; Holt-D is Holt's model with exponential smoothing; HOLT-W is the linear no trend Holt-Winters model; ARARMA is a long memory model; SES is the linear no trend simple exponential smoothing model. The RW is the random walk model with no drift. Percentages in bold at a forecast horizon is the models with lowest mean absolute percentage error.

Table 4

Median absolute percentage error for M3 monthly series

\begin{tabular}{|c|c|c|c|c|}
\hline \multirow[t]{2}{*}{ Forecast method } & \multicolumn{4}{|c|}{ Forecast horizon } \\
\hline & 1 & 6 & 12 & 18 \\
\hline RT & 0.116 & 0.152 & 0.179 & 0.199 \\
\hline ARIMA & 0.005 & 0.044 & 0.103 & 0.137 \\
\hline HOLT & 0.006 & 0.027 & 0.060 & 0.083 \\
\hline HOLT-D & 0.007 & 0.048 & 0.091 & 0.172 \\
\hline HOLT-W & 0.006 & 0.029 & 0.075 & 0.123 \\
\hline ARARMA & 0.999 & 1.006 & 1.029 & 1.033 \\
\hline SES & 0.033 & 0.105 & 0.164 & 0.232 \\
\hline RW & 0.007 & 0.066 & 0.120 & 0.197 \\
\hline
\end{tabular}

Note: RT is the robust trend model; ARIMA is the autoregressive integrated moving average model; HOLT is Holt's linear no trend model; Holt-D is Holt's model with exponential smoothing; HOLT-W is the linear no trend Holt-Winters model; ARARMA is a long memory model; SES is the linear no trend simple exponential smoothing model. The RW is the random walk model with no drift. Percentages in bold at a forecast horizon is the models with lowest median absolute percentage error.

The MdAPE statistic in Fig. 10 indicates the Holt model and Holt-W model typically forecast best for all horizons. The only exception is the ARIMA model forecast better than the Holt-W model at 12-periods ahead. The PB statistic in Fig. 11 shows the Holt-D model and ARIMA model are the best forecast models for most forecast horizons, with the Holt-W model is equally accurate in forecasting horizons 6-periods and 9periods ahead. That is, the MAPE, MdAPE and PB statistics suggest the Holt and Holt-D models provide an improvement over other linear extrapolation techniques and a random walk model in providing forecasts for monthly data. This indicates the Holt and Holt-D models are useful for establishing relatively accurate judgement-free projections of telecommunications data up to 18-periods ahead for monthly data. Holt and Holt-D models are superior for most forecast horizons. For short-horizon forecasts, the ARIMA model is best.

Tables 6 and 7 present the MAPE and MdAPE results of the out-of-sample forecasts for the 'other' series. The results in Tables 6 and 7 are similar to the forecast error results of the monthly series. The MAPE statistic in Table 6 shows that the Holt model is best forecast method for medium and long horizons, and yields lowest percentage error when forecasting 4-periods, 6-periods and 8-periods ahead. For short horizons of 1-period ahead, the MAPE statistic shows ARIMA and the Holt-D are the best for forecasting 'other' series. 
Table 5

Percent better for M3 monthly series

\begin{tabular}{|c|c|c|c|c|}
\hline \multirow[t]{2}{*}{ Forecast method } & \multicolumn{4}{|c|}{ Forecast horizon } \\
\hline & 1 & 6 & 12 & 18 \\
\hline RT & 1.400 & 10.345 & 6.897 & 44.828 \\
\hline ARIMA & 75.862 & 72.414 & 79.310 & 79.310 \\
\hline HOLT & 55.172 & 72.414 & 72.414 & 72.414 \\
\hline HOLT-D & 55.172 & 75.862 & 79.310 & 75.862 \\
\hline HOLT-W & 51.724 & 72.414 & 72.414 & 68.966 \\
\hline ARARMA & 13.793 & 3.448 & 10.345 & 10.345 \\
\hline SES & 3.448 & 3.448 & 6.897 & 6.897 \\
\hline
\end{tabular}

Note: RT is the robust trend model; ARIMA is the autoregressive integrated moving average model; HOLT is Holt's linear no trend model; Holt-D is Holt's model with exponential smoothing; HOLT-W is the linear no trend Holt-Winters model; ARARMA is a long memory model; SES is the linear no trend simple exponential smoothing model. The RW is not shown as the PB statistic shows the proportion of series for each model at each forecast horizon that is better than the forecasts of the RW model. Percentages in bold at a forecast horizon is the models with highest percentage accuracy.

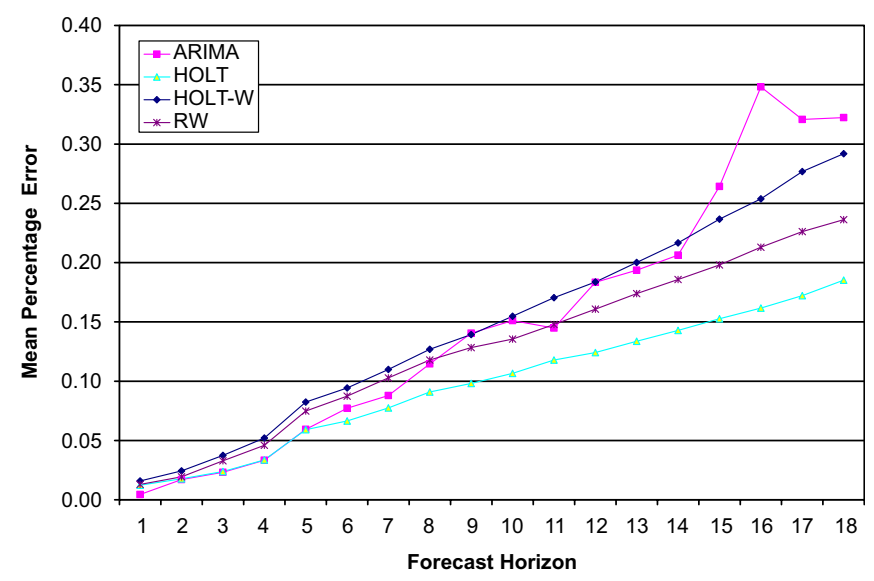

Fig. 9. Mean absolute percentage error for M3 monthly series.

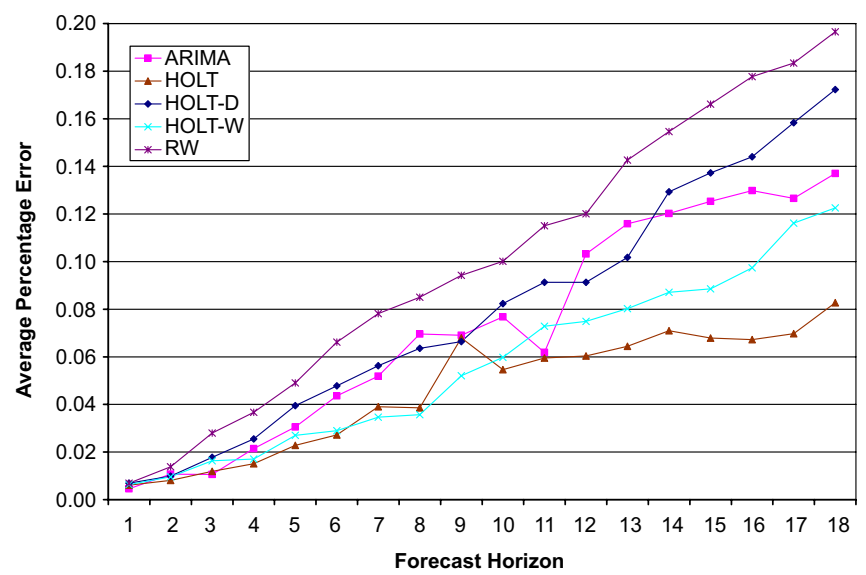

Fig. 10. Median absolute percentage error for M3 monthly series. 


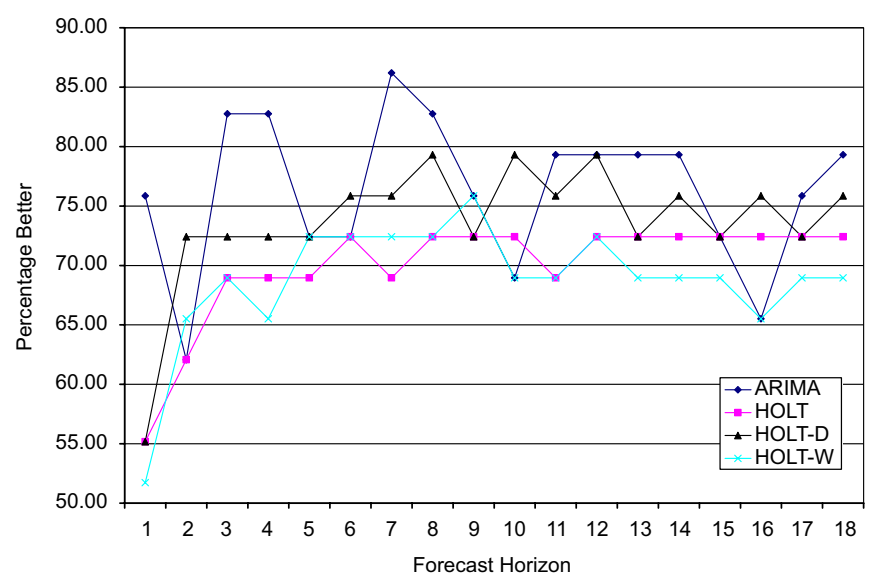

Fig. 11. Percentage better for M3 monthly series.

Table 6

Mean absolute percentage error for M3 'Other' series

\begin{tabular}{|c|c|c|c|c|}
\hline \multirow[t]{2}{*}{ Forecast method } & \multicolumn{4}{|c|}{ Forecast horizon } \\
\hline & 1 & 4 & 6 & 8 \\
\hline RT & 0.079 & 0.084 & 0.086 & 0.097 \\
\hline ARIMA & 0.009 & 0.034 & 0.051 & 0.071 \\
\hline HOLT & 0.010 & 0.025 & 0.036 & 0.049 \\
\hline HOLT-D & 0.009 & 0.030 & 0.047 & 0.070 \\
\hline HOLT-W & 0.012 & 0.032 & 0.047 & 0.064 \\
\hline ARARMA & 0.978 & 0.981 & 0.985 & 0.994 \\
\hline SES & 0.040 & 0.074 & 0.096 & 0.128 \\
\hline RW & 0.014 & 0.047 & 0.069 & 0.100 \\
\hline
\end{tabular}

Note: RT is the robust trend model; ARIMA is the autoregressive integrated moving average model; HOLT is Holt's linear no trend model; Holt-D is Holt's model with exponential smoothing; HOLT-W is the linear no trend Holt-Winters model; ARARMA is a long memory model; SES is the linear no trend simple exponential smoothing model. The RW is the random walk model with no drift. Percentages in bold at a forecast horizon is the models with lowest mean absolute percentage error.

Table 7

Median absolute percentage error for M3 'Other' series

\begin{tabular}{lllll}
\hline Forecast method & \multicolumn{2}{l}{ Forecast horizon } & & \\
\cline { 2 - 5 } & 1 & 4 & 0 & 8 \\
\hline RT & 0.070 & 0.073 & 0.079 & 0.041 \\
ARIMA & 0.006 & 0.028 & $\mathbf{0 . 0 2 7}$ & 0.058 \\
HOLT & 0.008 & $\mathbf{0 . 0 2 2}$ & 0.036 & 0.033 \\
HOLT-D & $\mathbf{0 . 0 0 6}$ & $\mathbf{0 . 0 2 2}$ & $\mathbf{0 . 0 3 3}$ \\
HOLT-W & 0.009 & $\mathbf{0 . 0 2 2}$ & 1.001 & 0.050 \\
ARARMA & 1.002 & 0.062 & 0.089 & 1.016 \\
SES & 0.037 & 0.040 & 0.062 & 0.119 \\
RW & 0.012 & & 0.089
\end{tabular}

Note: RT is the robust trend model; ARIMA is the autoregressive integrated moving average model; HOLT is Holt's linear no trend model; Holt-D is Holt's model with exponential smoothing; HOLT-W is the linear no trend Holt-Winters model; ARARMA is a long memory model; SES is the linear no trend simple exponential smoothing model. The RW is the random walk model with no drift. Percentages in bold at a forecast horizon is the models with lowest median absolute percentage error. 
The MdAPE error statistic presented in Table 7 shows a similar result to the MAPE statistic. The Holt model is the best for forecasting 4-periods, 6-periods and 8-periods ahead. The MdAPE statistic also shows the Holt-D model is best for forecasting short- and intermediate-horizon periods, viz., 1-period and 4-periods ahead. An exception contained in Table 7 shows the Holt-W model performs well as the Holt and Holt-D models when forecasting 4-periods ahead.

The PB statistic presented in Table 8 shows the Holt-D model is best for forecasting short- and intermediate-horizons of 1-period and 4-periods ahead for the 'other' series, while the Holt model is best suited for forecasting the intermediate to long-horizons of 6-periods and 8-periods ahead.

When forecasting the 'other' series, the MAPE and MdAPE statistics contained in Figs. 12 and 13 show that the Holt, Holt-D and Holt-W methods provide an improvement over other linear extrapolation techniques and random walk model. The PB statistic in Fig. 14 shows that the Holt model and Holt-D models provide better forecasts for the 'other' series. The results suggest that the Holt model, Holt-D model and Holt-W models are useful for establishing relatively accurate judgement-free projections of telecommunications data of up to 8-periods ahead for telecommunications data of unknown periodicity. The results of the Holt and Holt-D models are superior for most horizons.

Table 8

Percent better for M3 'Other' series

\begin{tabular}{|c|c|c|c|c|}
\hline \multirow[t]{2}{*}{ Forecast method } & \multicolumn{4}{|c|}{ Forecast horizon } \\
\hline & 1 & 4 & 6 & 8 \\
\hline RT & 1.667 & 9.167 & 17.500 & 48.333 \\
\hline ARIMA & 65.833 & 67.500 & 68.333 & 70.000 \\
\hline HOLT & 65.000 & 75.000 & 76.667 & 79.167 \\
\hline HOLT-D & 69.167 & 75.833 & 75.833 & 76.667 \\
\hline HOLT-W & 59.167 & 70.000 & 69.167 & 73.333 \\
\hline ARARMA & 1.667 & 0.833 & 1.667 & 1.667 \\
\hline SES & 6.667 & 5.833 & 5.000 & 5.833 \\
\hline
\end{tabular}

Note: RT is the robust trend model; ARIMA is the autoregressive integrated moving average model; HOLT is Holt's linear no trend model; Holt-D is Holt's model with exponential smoothing; HOLT-W is the linear no trend Holt-Winters model; ARARMA is a long memory model; SES is the linear no trend simple exponential smoothing model. The RW is not shown as the PB statistic shows the proportion of series for each model at each forecast horizon that is better than the forecasts of the RW model. Percentages in bold at a forecast horizon is the models with highest percentage accuracy.

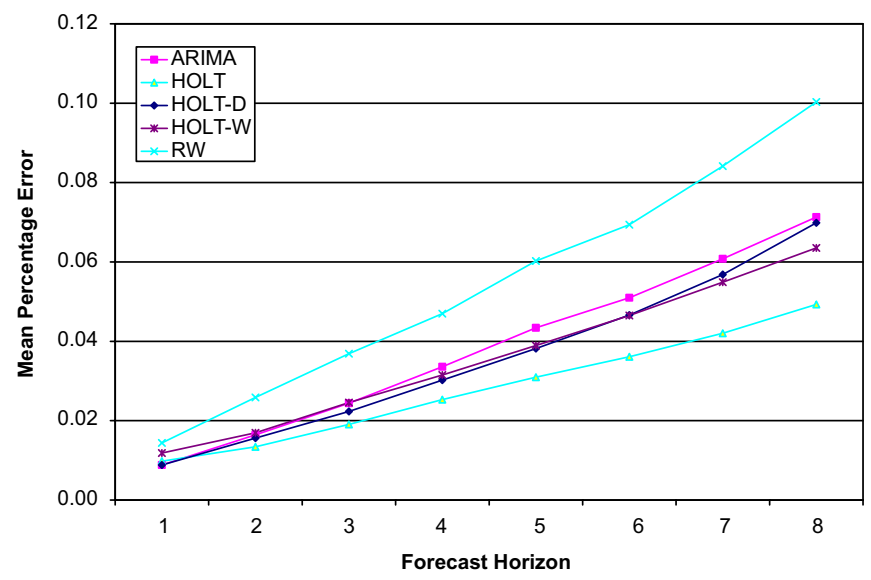

Fig. 12. Mean absolute percentage error for M3 'Other' series. 


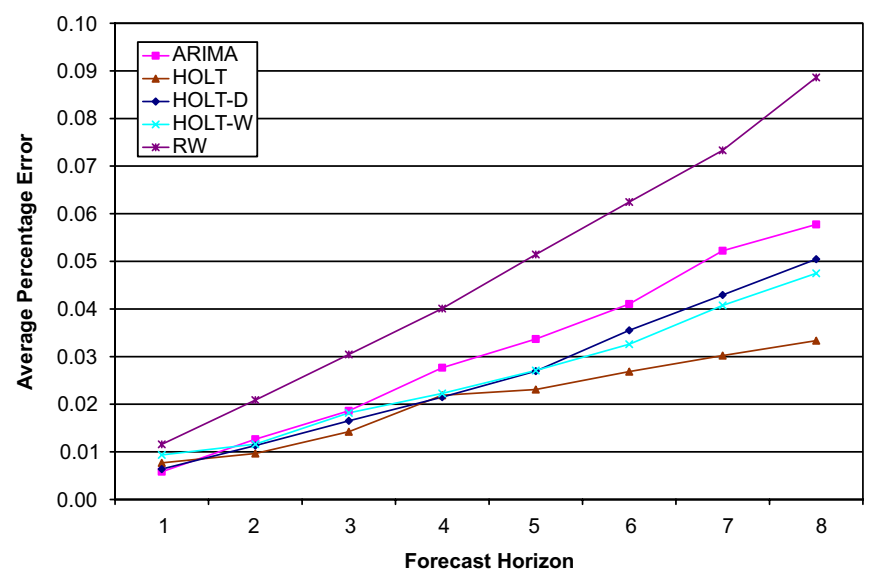

Fig. 13. Median absolute percentage error for M3 'Other' series.

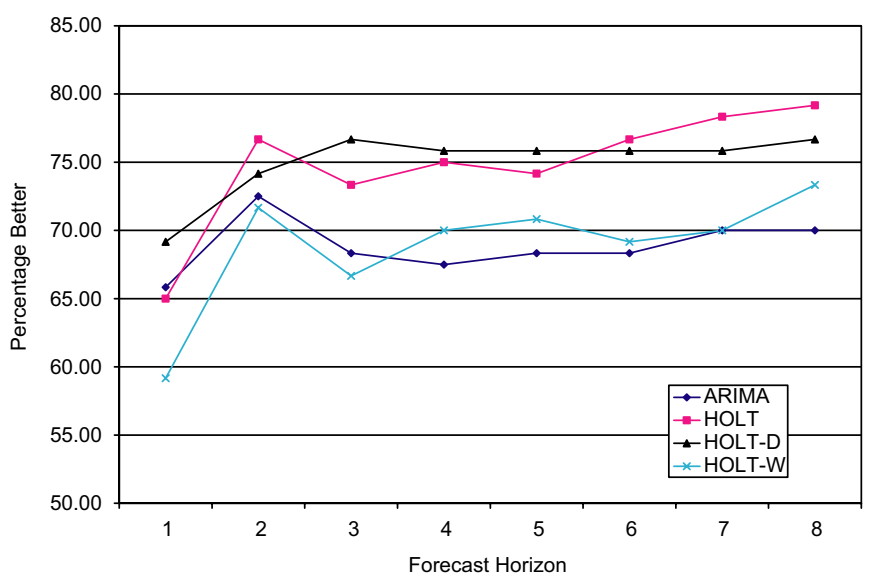

Fig. 14. Percentage better for M3 'Other' series.

A summary of the results by error statistic and forecast horizon are presented in Tables 9-11. The tables indicate that the best model for forecasting telecommunication series without any domain knowledge for the forecast horizons tested are the ARIMA, Holt and the Holt-D models.

\section{Conclusion}

This analysis intends to identify those of the linear models proposed in ITU's Recommendation E.507 (to aid telecommunication companies in forecasting) provide the better forecasts when little domain information is available. In particular, the analysis covers situation whereby little or no information is available about the reliability or quality of data. Forecasts from the ARARMA, ARIMA and several SES model specifications are compared against those for a random walk model. The results show despite having no knowledge of the origin of the telecommunications series, the models can accurately forecast telecommunications data. This suggests imperfections that may be inherent within telecommunications data might not unduly affect the accuracy of generated telecommunication forecasts. Telecommunications data are often not well understood and are short in length. Hence, the use of these univariate models without assumptions before may be most appropriate. The study shows the linear extrapolation models perform well when used to forecast monthly and 'other' M3 telecommunications series of unknown periodicity. The sample forecast accuracy based on MAPE and MdAPE statistics, and PB measure show the Holt and Holt-D models provides the most reliable forecasts 
Table 9

Best models for forecasting M3 series by MAPE

\begin{tabular}{|c|c|c|c|c|}
\hline \multirow[t]{2}{*}{ M3 series } & \multicolumn{4}{|l|}{ Forecast horizon } \\
\hline & 1 & 6 & 12 & 18 \\
\hline Monthly & ARIMA & HOLT & HOLT & HOLT \\
\hline Other & ARIMA/HOLT-D & HOLT & HOLT & HOLT \\
\hline
\end{tabular}

Note: RT is the robust trend model; ARMA is the autoregressive integrated moving.

Table 10

Best models for forecasting M3 series by MdAPE

\begin{tabular}{lllll}
\hline M3 series & Forecast horizon & & \\
\cline { 2 - 5 } & 1 & 6 & 12 & 18 \\
\hline Monthly & ARIMA & HOLT-D & HOLT-D & HOLT \\
Other & HOLT-D & HOLT/HOLT-D/HOLT-W & HOLT \\
\hline
\end{tabular}

Note: RT is the robust trend model; ARMA is the autoregressive integrated moving.

Table 11

Best models for forecasting M3 series by PB

\begin{tabular}{lllll}
\hline M3 series & \multicolumn{2}{l}{ Forecast horizon } & & \\
\cline { 2 - 5 } & 1 & 6 & HOLT/HOLT-D & 12 \\
\hline Monthly & ARIMA & HOLT-D & HOLT & HOLT-D \\
Other & HOLT-D & HOLT-D & HOLT \\
\hline
\end{tabular}

Note: RT is the robust trend model; ARMA is the autoregressive integrated moving.

without any knowledge of the underlying dynamics of the telecommunications data series, an outcome similar to the recommendations of the M3-competition. The results suggest that further gains in forecast accuracy may not be forthcoming through the deployment of more sophisticated nonlinear models, as the forecast performance of the univariate linear models applied can generate more accurate forecasts than the random walk for intermediate and long-horizons future periods.

\section{Appendix}

To estimate the MAPE for method $i$ and horizon $h$ for series $j$, the absolute percentage error $A P E_{i, h, j}$ is first calculated:

$$
A P E_{i, h, j}=\left|\frac{F_{i, h, j}-A_{h, j}}{A_{h, j}}\right|,
$$

where $F_{i, h, j}$ is the forecast for method $i$ for horizon $h$ using series $j$ and $A_{h, j}$ is the actual value at horizon $h$ for series $j$. The $M A P E_{i, h, j}$ is then calculated by finding the mean of the $A P E_{i, h, j}$ series:

$$
M A P E_{i, h, j}=\operatorname{mean}\left(A P E_{i, h, j}\right) \text {. }
$$

As none of the series observations are negative, the MAPE is appropriate. An advantage of applying the MAPE is that it is scale-invariant. To estimate the MdAPE error statistic for method $i$ for series $j$ from the 
$A P E_{i, h, j}$ error statistic, the median of the $A P E_{i, h, j}$ series is calculated:

$$
M d R A E=\operatorname{median}\left(A P E_{i, h, j}\right),
$$

where $s$ is the total number of total number of series forecasted and MdAPE is observation $(s+1) / 2$ if $s$ is odd, or the mean of observations $s / 2$ when $s$ is even when $A P E_{i, h, j}$ observations are ordered by rank. The PB statistic counts the proportion a given method has a forecasting error larger than a relative method:

$$
P B_{i, h, j}=\left[\frac{1}{s} \sum_{j=1}^{s} \delta_{i, h, j}\right] 100,
$$

where

$$
\delta_{i, h, j}= \begin{cases}1 & \text { if }\left|F_{i, h, j}-A_{h, j}\right| \prec\left|F_{r w, h, j}-A_{h, j}\right| \\ 0 & \text { otherwise. }\end{cases}
$$

$F_{i, h, j}$ is the forecast for method $i$ at horizon $h$ for series $j$ and $A_{h, j}$ is the actual value at horizon $h$ for series $i$. $\delta_{i, h, j}$ is a dummy variable that records the proportion of times a particular model $i$ forecasting horizon $h$ for series $j$ has a lower forecast error than the random walk model and $s$ is the total number of series forecasted and $P B_{i, h, j}$ is the proportion (in percentage) method $i$ performs better that the relative method. The relative method applied for comparison in this study is the random walk model. A value of greater than $50 \%$ for $P B_{i, h, j}$ indicates the forecasts obtained for a particular forecasting method $i$ is more accurate than the random walk.

\section{References}

Armstrong, J., \& Collopy, F. (1992). Error measures for generalizing about forecast methods: Empirical comparisons. International Journal of Forecasting, 8, 69-80.

Fildes, R. (1992). The evaluation of extrapolative forecasting methods. International Journal of Forecasting, 8, 81-98.

Fildes, R., Hibon, M., Makridakis, S., \& Meade, N. (1998). Generalising about univariate forecasting methods: Further empirical evidence. International Journal of Forecasting, 14, 339-358.

Grambsch, P., \& Stahel, W. (1990). Forecasting demand for special services. International Journal of Forecasting, 6, 53-64.

Grubesic, T., \& Murray, A. (2005). Geographies of imperfection in telecommunication analysis. Telecommunications Policy, $29,69-94$.

Makridakis, S., Chatfield, C., Hibon, M., Lawrence, M., Mills, T., Ord, K., et al. (1993). The M-2 competition: A real-time judgmentally based forecasting study. International Journal of Forecasting, 9, 5-23.

Makridakis, S., \& Hibon, M. (2000). The M3-competition: Results, conclusions and implications. International Journal of Forecasting, 16, 451-476.

Parzen, E. (1982). ARARMA models for time series analysis and forecasting. Journal of Forecasting, 1, 67-82. 